This item was submitted to Loughborough's Research Repository by the author.

Items in Figshare are protected by copyright, with all rights reserved, unless otherwise indicated.

\title{
Breakdown of the Wiedemann-Franz law in strongly-coupled electron- phonon system, application to the cuprates
}

PLEASE CITE THE PUBLISHED VERSION

LICENCE

CC BY-NC-ND 4.0

\section{REPOSITORY RECORD}

Lee, K.K., A.S. Alexandrov, and W.Y. Liang. 2019. "Breakdown of the Wiedemann-franz Law in Stronglycoupled Electron-phonon System, Application to the Cuprates". figshare. https://hdl.handle.net/2134/1218. 


\title{
Breakdown of the Wiedemann-Franz law in strongly-coupled electron-phonon system, application to the cuprates
}

\author{
K. K. Lee, ${ }^{1}$ A. S. Alexandrov ${ }^{2}$ and W. Y. Liang ${ }^{1}$ \\ ${ }^{1}$ IRC in Superconductivity, Cavendish Laboratory, \\ University of Cambridge, Cambridge, CB3 OHE, United Kingdom \\ ${ }^{2}$ Department of Physics, Loughborough University, Loughborough LE11 3TU, United Kingdom
}

\begin{abstract}
With the superconducting cuprates in mind, a set of unitary transformations was used to decouple electrons and phonons in the strong-coupling limit. While phonons remain almost unrenormalised, electrons are transformed into itinerent singlet and triplet bipolarons and thermally excited polarons. The triplet/singlet exchange energy and the binding energy of the bipolarons are thought to account for the spin and charge pseudogaps in the cuprates, respectively. We calculated the Hall Lorenz number of the system to show that the Wiedemann-Franz law breaks down due to the interference of the polaron and bipolaron contributions to heat flow. The model provides a quantitative fit to magnetotransport data in the cuprates. Furthermore we are able to extract the phonon component of the thermal conductivity with the use of experimental data and the model. Our results further validate the use of a charged Bose gas model to describe normal and superconducting properties of unconventional superconductors.
\end{abstract}

\section{INTRODUCTION}

The theory of high temperature superconductivity is one of the biggest challenges in condensed matter physics today and is reflected by the large number of the theoretical models 1.2 .3 .4 .5 .6 proposed to date. Most of these theories have a foundation built on the unconventional Cooper pairs whilst others concentrate on the basic principals of the symmetry breaking and competing orders. On the other hand the electronphonon interaction continue to gather support through isotope effect measurements ${ }^{7}$, infrared ${ }^{8.9 .10}$ and thermal conductivity 11 , neutron scattering ${ }^{12}$, and more recently in ARPES13.14. To account for the high values of $\mathrm{T}_{c}$ in the cuprates, it is necessary to have electron-phonon interactions larger than those found in the intermediate coupling theory of superconductivity $\underline{15}$. Regardless of the adiabatic ratio, the Migdal-Eliashberg theory of superconductivity and Fermi-liquids has been shown to breakdown at $\lambda=1^{16}$ using the $(1 / \lambda)$ expansion technique $\mathrm{in}^{17}$. The many-electron system collapses into the small (bi)polaron regime $\frac{16,18.19}{\text { at }} \lambda \geq 1$ with well separated vibration and charge-carrier degrees of freedom.

Remarkably the discovery of high $\mathrm{T}_{c}$ cuprates was motivated by the polaron model ${ }^{20}$. The isotope effect, high values of the static dielectric constants and optical spectroscopy in the cuprates suggest that the electron-phonon interaction is sufficient to bind small polarons into small bipolarons. At first sight these carriers have a mass too large to be mobile, however it has been shown that the inclusion of the on-site Coulomb repulsion leads to the favoured binding of intersite oxygen holes ${ }^{2.21}$. The intersite bipolarons can then tunnel with an effective mass of only 10 electron masses 21.22 .23 .24 .

Mott and one of the authors (ASA) proposed a simple model $^{25}$ of the cuprates based on bipolarons, Fig.1. In this model all the holes (polarons) are bound into small intersite singlet and triplet bipolarons at any tempera- ture. Above $\mathrm{T}_{c}$ this Bose gas is non-degenerate and below $\mathrm{T}_{c}$ phase coherence (ODLRO) of the preformed bosons sets in, followed by superfluidity of the charged carriers. The triplet and singlet states are separated by an exchange energy J which explains the spin gap observed in many NMR and neutron scattering experiments ${ }^{26.27}$. Of course, there are also thermally excited single polarons in the model. Their density becomes comparable with the bipolaron density at the temperature $T^{*}$ which is about half of the bipolaron binding energy $\Delta$. There is much evidence for the crossover regime at $T^{*}$ and normal state pseudogap in the cuprates ${ }^{28}$. For example, measurements of single particle tunneling and Andreev reflections ${ }^{29}$ clearly demonstrate that there exists two distinct energy gaps. These energy gaps are half of the binding energy of preformed bipolarons and the coherence energy gap of the superconducting phase as proposed in Ref ${ }^{30}$.

Other experimental observations which have been satisfactorily explained using this particular approach include Hall ratio ${ }^{31}$, Hall angle ${ }^{31}$, in-plane resistivity ${ }^{31}$, infrared conducitivity ${ }^{32}$, magnetic susceptibility ${ }^{33}$, tunneling spectroscopy ${ }^{34}$, isotope effect $^{35}$, thermal conducitivity $^{36}$ and the upper critical field ${ }^{37}$ in the superconducting state. ARPES measurements indicate the presence of an angular dependent narrow peak and a featureless background. In the polaronic model the ARPES spectrum can be explained if one considers a charge transfer Mott-insulator and the single polaron spectral function ${ }^{38}$. The conclusion is that the ARPES spectrum can in fact be explained with the absence or with a small Fermi surface. Specific heat measurements have a striking resemblance to the superfluid transition of weakly-interacting 3D bosons 39 . A theoretical mode $\stackrel{40}{ }$, experimental data and analysis of the specific heat in a magnetic field ${ }^{41}$ suggest this is a convincing argument.

In this paper we develop the theory of the normal state magnetotransport in bipolaronic systems and explain the 


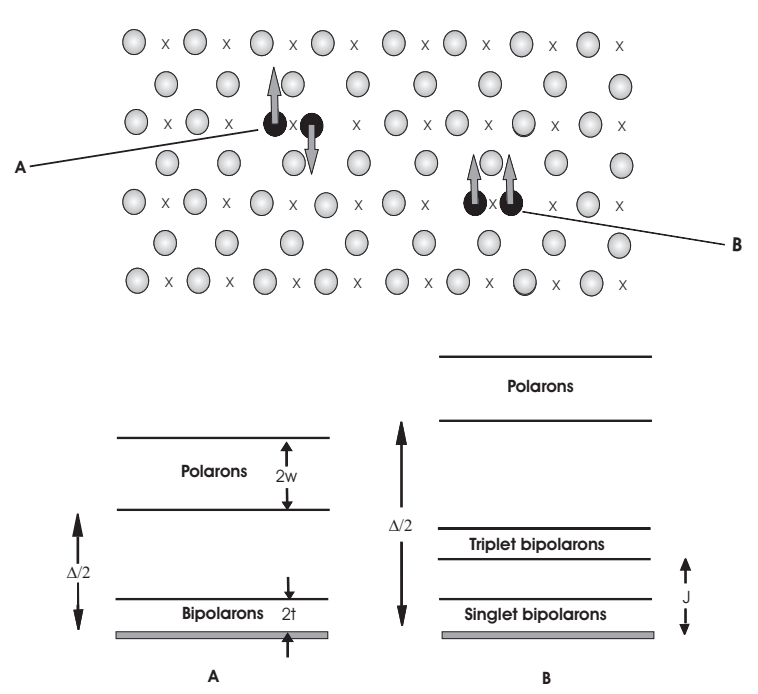

FIG. 1: Bipolaron picture of high temperature superconductors. $A$ corresponds to the singlet intersite bipolaron. $B$ is the triplet intersite bipolaron, which naturally includes the addition of an extra excitation band. The crosses are copper sites and the circles are oxygen sites. w is the half bandwidth of the polaron band, $\mathrm{t}$ is the half bandwidth of the bipolaron band, $\Delta / 2$ is the bipolaron binding energy per polaron and $\mathrm{J}$ is the exchange energy per bipolaron.

experimental Hall Lorenz number for $\mathrm{YBa}_{2} \mathrm{Cu}_{3} \mathrm{O}_{6+x}$ as measured by Zhang et al. ${ }^{42}$. The case of degenerate singlet and triplet bipolarons was briefly discussed by us in Ref ${ }^{43}$. Particular interest within this paper lies in the conclusion that the Wiedemann-Franz law is violated in cuprates. This departure from the Fermi liquid picture is seen in both the superconducting and normal state and might be related by a common mechanism 44 .

The extraction of the electronic thermal conductivity term has proven difficult as both the electronic term, $\kappa_{e l}$ and the phononic term, $\kappa_{p h}$ make a comparable contribution. However recent experiments appear to have got around this problem ${ }^{42.44 .45}$. Takenaka et al $\stackrel{44}{=}$ found that the insulating state thermal conductivity (therefore predominantly phononic) was approximately independent of doping except at y $\sim 1-0.75$ in $\mathrm{YBa}_{2} \mathrm{Cu}_{3} \mathrm{O}_{7-y}$. Therefore the phononic term for the thermal conductivity above $y \sim 0.5$ could be estimated. This analysis led to the conclusion that the $\kappa_{e l}$ is constant or weakly Tdependent in the normal state like that found by Salamon et $\mathrm{a}^{45}$. This approximately T-independent $\kappa_{e l}$ in the underdoped region therefore implies the violation of the Wiedemann-Franz law (since the resistivity is found to be a non-linear function of temperature in this regime). This breakdown of the Wiedemann-Franz law has been seen in other cuprates such as $\mathrm{Pr}_{2-x} \mathrm{Ce}_{x} \mathrm{CuO}_{4}$ at optimal doping 46 and $\mathrm{La}_{2-x} \mathrm{Sr}_{x} \mathrm{CuO}_{4}$ at underdoping 47 . Zhang et $a^{142}$ recently developed a novel method to determine the Lorenz number, based on the thermal Hall conductivity. The thermal Hall effect is a purely electronic effect since phonons are not affected by the mag- netic field. As a result, the "Hall" Lorenz number, $L_{H}=\left(e / k_{B}\right)^{2} \kappa_{x y} /\left(T \sigma_{x y}\right)$, has been directly measured in $\mathrm{YBa}_{2} \mathrm{Cu}_{3} \mathrm{O}_{6.95}$ because transverse thermal $\kappa_{x y}$ and electrical $\sigma_{x y}$ conductivities involve only the electrons. The experimental $L_{x y}$ showed a linear temperature dependence, which violates the Wiedemann-Franz law. It is clear that it would be difficult to explain this experimental observation in the framework of any Fermi-liquid model.

A charge 2e Bose gas of bipolarons naturally leads to the conclusion ${ }^{36}$ that the Lorenz number in the normal state is strongly suppressed due to the double charge per carrier and classical statistics of bipolarons compared with the Lorenz number in the normal metal, $L_{0}=\pi^{2} / 3$. Here we demonstrate that the WiedemannFranz law breaks down because of the interference of the polaron and bipolaron contributions to the heat transport. When thermally excited polarons and triplet pairs are included, the bipolaron model explains the violation of the Wiedemann-Franz law in the cuprates and the Hall Lorenz number as seen in the experiments.

\section{LOW FERMI ENERGY: PAIRING IS INDIVIDUAL IN MANY CUPRATES}

The possibility of real-space pairing, as opposed to BCS-like pairing, has been the subject of much discussion. Experimental and theoretical evidence for an exceptionally strong electron-phonon interaction in high temperature superconductors is now so overwhelming, that even advocates of nonphononic mechanisms $\frac{48}{3}$ accept this fact. Nevertheless the same authors 3.48 dismiss any real-space pairing claiming that pairing is collective in cuprates. They believe in a large Fermi surface with the number of holes $(1+x)$ rather than $x$ in superconducting cuprates, where $x$ is the doping level like in $\mathrm{La}_{2-x} \mathrm{Sr}_{x} \mathrm{CuO}_{4}$. As an alternative to a three-dimensional Bose-Einstein condensation of bipolarons these authors suggest a collective pairing (i.e the Cooper pairs in the momentum space) at some temperature $T^{*}>T_{c}$, but without phase ordering. In this concept the phase coherence and superconducting critical temperature $T_{c}$ are determined by the superfluid density, which is proportional to doping $x$ due to a low dimensionality, rather then to the total density of carriers $(1+x)$. On the experimental side a large Fermi surface is clearly incompatible with a great number of thermodynamic, magnetic, and kinetic measurements, which show that only holes doped into a parent insulator are carriers in the normal state. On the theoretical ground this preformed Cooper-pair (or phase-fluctuation) scenario contradicts a theorem ${ }^{49}$, which proves that the number of supercarriers (at $T=0)$ and normal-state carriers is the same in any clean superfluid.

Objections against real-space pairing also contradict a parameter-free estimate of the Fermi energy, which shows that the pairing is individual in cuprates ${ }^{50}$. Indeed, first- 
principles band structure calculations show that copper, alkali metal, and magnesium donate their outer electrons to oxygen, $C_{60}$, and boron in cuprates, doped fullerenes, and in $M g B_{2}$, respectively. In cuprates the band structure is quasi-two-dimensional (2D) with a few degenerate hole pockets. Applying the parabolic approximation for the band dispersion we obtain the renormalized Fermi energy as

$$
\epsilon_{F}=\frac{\hbar^{2} \pi n_{i} d}{m_{i}^{*}},
$$

where $d$ is the interplane distance, and $n_{i}, m_{i}^{*}$ are the density of holes and their effective mass in each of the hole subbands $i$ renormalized by the electron-phonon (and by any other) interaction. One can express the renormalized band-structure parameters through the in-plane magnetic-field penetration depth at $T \approx 0$, measured experimentally:

$$
\lambda_{H}^{-2}=4 \pi e^{2} \sum_{i} \frac{n_{i}}{m_{i}^{*} c^{2}} .
$$

As a result, we obtain a parameter-free expression for the "true" Fermi energy as

$$
\epsilon_{F}=\frac{\hbar^{2} d c^{2}}{4 g e^{2} \lambda_{H}^{2}},
$$

where $g$ is the degeneracy of the spectrum which might depend on doping in cuprates. One expects 4 hole pockets inside the Brillouin zone (BZ) due to the MottHubbard gap in underdoped cuprates. If the hole band minima are shifted with doping to BZ boundaries, all their wave vectors would belong to the stars with two or more prongs. The groups of wave vectors for these stars have only $1 \mathrm{D}$ representations. It means that the spectrum will be degenerate with respect to the number of prongs which the star has, i.e $g \geqslant 2$. Because Eq.(3) does not contain any other band-structure parameters, the estimate of $\epsilon_{F}$ using this equation does not depend very much on the parabolic approximation for the band dispersion.

Generally, the ratios $n / m^{*}$ in Eq.(1) and Eq.(2) are not necessarily the same. The 'superfluid' density in Eq.(2) might be different from the total density of delocalized carriers in Eq.(1). However, in a translationally invariant system they must be the same ${ }^{49}$. This is also true even in the extreme case of a pure two-dimensional superfluid, where quantum fluctuations are important. One can, however, obtain a reduced value of the zero temperature superfluid density in the dirty limit, $l \ll \xi(0)$, where $\xi(0)$ is the zero-temperature coherence length. The latter was measured directly in cuprates as the size of the vortex core. It is about $10 \stackrel{\circ}{A}$ or even less. On the contrary, the mean free path was found surprisingly large at low temperatures, $l \sim 100-1000 \AA$. Hence, it is rather probable that novel superconductors are in the clean limit, $l \gg \xi(0)$, so that the parameter-free expression for $\epsilon_{F}$, Eq.(3) holds.

\begin{tabular}{|c|c|c|c|c|}
\hline Compound & $T_{c}(\mathrm{~K})$ & $\lambda_{H, a b}(\stackrel{\AA}{A})$ & $\mathrm{d}(\stackrel{\circ}{A})$ & $g \epsilon_{F}(\mathrm{meV})$ \\
\hline $\mathrm{La}_{1.8} \mathrm{Sr}_{0.2} \mathrm{CuO} \mathrm{O}_{4}$ & 36.2 & 2000 & 6.6 & 112 \\
\hline $\mathrm{La}_{1.78} \mathrm{Sr}_{0.22} \mathrm{CuO}_{4}$ & 27.5 & 1980 & 6.6 & 114 \\
\hline $\mathrm{La}_{1.76} \mathrm{Sr}_{0.24} \mathrm{CuO}_{4}$ & 20.0 & 2050 & 6.6 & 106 \\
\hline $\mathrm{La}_{1.85} \mathrm{Sr}_{0.15} \mathrm{CuO}_{4}$ & 37.0 & 2400 & 6.6 & 77 \\
\hline $\mathrm{La}_{1.9} \mathrm{Sr}_{0.1} \mathrm{CuO}_{4}$ & 30.0 & 3200 & 6.6 & 44 \\
\hline $\mathrm{La}_{1.75} \mathrm{Sr}_{0.25} \mathrm{CuO}_{4}$ & 24.0 & 2800 & 6.6 & 57 \\
\hline $\mathrm{YBa}_{2} \mathrm{Cu}_{3} \mathrm{O}_{7}$ & 92.5 & 1400 & 4.29 & 148 \\
\hline$Y B a C u O(2 \% Z n)$ & 68.2 & 2600 & 4.29 & 43 \\
\hline$Y B a C u O(3 \% Z n)$ & 55.0 & 3000 & 4.29 & 32 \\
\hline$Y B a C u O(5 \% Z n)$ & 46.4 & 3700 & 4.29 & 21 \\
\hline $\mathrm{YBa}_{2} \mathrm{Cu}_{3} \mathrm{O}_{6.7}$ & 66.0 & 2100 & 4.29 & 66 \\
\hline $\mathrm{YBa}_{2} \mathrm{Cu}_{3} \mathrm{O}_{6.57}$ & 56.0 & 2900 & 4.29 & 34 \\
\hline $\mathrm{YBa}_{2} \mathrm{Cu}_{3} \mathrm{O}_{6.92}$ & 91.5 & 1861 & 4.29 & 84 \\
\hline$Y \mathrm{Ba}_{2} \mathrm{Cu}_{3} \mathrm{O}_{6.88}$ & 87.9 & 1864 & 4.29 & 84 \\
\hline$Y \mathrm{Ba}_{2} \mathrm{Cu}_{3} \mathrm{O}_{6.84}$ & 83.7 & 1771 & 4.29 & 92 \\
\hline $\mathrm{YBa}_{2} \mathrm{Cu}_{3} \mathrm{O}_{6.79}$ & 73.4 & 2156 & 4.29 & 62 \\
\hline $\mathrm{YBa}_{2} \mathrm{Cu}_{3} \mathrm{O}_{6.77}$ & 67.9 & 2150 & 4.29 & 63 \\
\hline $\mathrm{YBa}_{2} \mathrm{Cu}_{3} \mathrm{O}_{6.74}$ & 63.8 & 2022 & 4.29 & 71 \\
\hline$Y \mathrm{Ba}_{2} \mathrm{Cu}_{3} \mathrm{O}_{6.7}$ & 60.0 & 2096 & 4.29 & 66 \\
\hline$Y \mathrm{Ba}_{2} \mathrm{Cu}_{3} \mathrm{O}_{6.65}$ & 58.0 & 2035 & 4.29 & 70 \\
\hline $\mathrm{YBa}_{2} \mathrm{Cu}_{3} \mathrm{O}_{6.6}$ & 56.0 & 2285 & 4.29 & 56 \\
\hline $\mathrm{HgBa}_{2} \mathrm{CuO}_{4.049}$ & 70.0 & 2160 & 9.5 & 138 \\
\hline $\mathrm{HgBa}_{2} \mathrm{CuO} \mathrm{O}_{4.055}$ & 78.2 & 1610 & 9.5 & 248 \\
\hline $\mathrm{HgBa}_{2} \mathrm{CuO} \mathrm{O}_{4.055}$ & 78.5 & 2000 & 9.5 & 161 \\
\hline $\mathrm{HgBa}_{2} \mathrm{CuO} \mathrm{O}_{4.066}$ & 88.5 & 1530 & 9.5 & 274 \\
\hline $\mathrm{HgBa}_{2} \mathrm{CuO}_{4.096}$ & 95.6 & 1450 & 9.5 & 305 \\
\hline $\mathrm{HgBa}_{2} \mathrm{CuO}_{4.097}$ & 95.3 & 1650 & 9.5 & 236 \\
\hline $\mathrm{HgBa}_{2} \mathrm{CuO}_{4.1}$ & 94.1 & 1580 & 9.5 & 257 \\
\hline $\mathrm{HgBa}_{2} \mathrm{CuO}_{4.101}$ & 93.4 & 1560 & 9.5 & 264 \\
\hline $\mathrm{HgBa}_{2} \mathrm{CuO}_{4.101}$ & 92.5 & 1390 & 9.5 & 332 \\
\hline $\mathrm{HgBa}_{2} \mathrm{CuO}_{4.105}$ & 90.9 & 1560 & 9.5 & 264 \\
\hline $\mathrm{HgBa}_{2} \mathrm{CuO}_{4.108}$ & 89.1 & 1770 & 9.5 & 205 \\
\hline
\end{tabular}

TABLE I: The Fermi energy (multiplied by the degeneracy) of cuprates

Parameter-free estimates of the Fermi energy obtained by using Eq.(3) are presented in the Table. The renormalised Fermi energy in more than 30 cuprates is definitely less than $100 \mathrm{meV}$.

In many cases (Table) the renormalized Fermi energy is so small that pairing is certainly individual. Such pairing will occur when the size of a pair, $\rho$ is smaller than the inter-pair separation, $r$. The size of a pair is generally

$$
\rho=\frac{\hbar}{\sqrt{m^{*} \Delta}},
$$

where $\Delta$ is its binding energy. The separation of pairs can be directly related to the Fermi energy in $2 \mathrm{D}$

$$
r=\hbar \sqrt{\frac{\pi}{\epsilon_{F} m^{*}}} .
$$


We see that the true condition for real-space pairing is

$$
\epsilon_{F} \lesssim \pi \Delta
$$

The bipolaron binding energy is thought to be twice the so-called pseudogap ${ }^{2}$. Experimentally measured pseudogap of many cuprates 28 is as large as $\Delta / 2 \gtrsim$ $50 \mathrm{meV}$, so that Eq.(6) is well satisfied in underdoped and even in a few optimally and overdoped cuprates. One should notice that the coherence length in the charged Bose gas has nothing to do with the size of the boson as erroneously assumed by some authors 48 . It depends on the interparticle distance and the mean-free path, and might be as large as in the BCS superconductor. Hence, it would be incorrect to apply the ratio of the coherence length to the inter-carrier distance as a criterium of the BCS-Bose liquid crossover. The correct criterium is given by Eq.(6).

\section{NON-EQUILIBRIUM BIPOLARON AND POLARON DISTRIBUTION FUNCTIONS}

Because thermally excited phonons and (bi)polarons are well decoupled in the strong-coupling regime of the electron-phonon interaction the standard Boltzmann equation for kinetics of renormalised carriers may be applied. The distribution function for each carrier is given as

$$
f=f_{0}+f_{1} .
$$

Here $f_{0}$ is the distribution function at equilibrium and $f_{1}(\mathbf{k})$ is the deviation of the distribution function away from equilibrium. We assume that $f_{1}$ is small compared to $f_{0} . f_{0}^{p}$ (polaron) is the Fermi-Dirac distribution and both $f_{0}^{s}$ and $f_{0}^{t}$ (singlet and triplet bipolaron, respectively) are the Bose-Einstein distributions,

$$
\begin{gathered}
f_{0}^{p}=\frac{1}{\exp [(E+\Delta / 2-\mu / 2) / T]+1}, \\
f_{0}^{s}=\frac{1}{\exp [(E-\mu) / T]-1}, \\
f_{0}^{t}=\frac{1}{\exp [(E+J-\mu) / T]-1} .
\end{gathered}
$$

$\mu$ is the chemical potential, $J$ is the exchange energy which separates the triplet state from the singlet state, and $\Delta$ is the bipolaron binding energy per pair which is assumed to be of s-symmetry. The latter assumption of s-wave bulk pairing symmetry of a single-particle gap has been shown to be a valid one $e^{30,51,52}$.

We make use of the $\tau$-approximation ${ }^{53}$ in the presence of the electric field $\mathbf{E}$, temperature gradient $\nabla T$ and magnetic field $\mathbf{B} \| \mathbf{z} \perp \mathbf{E}$ and $\nabla T$,

$$
f(\mathbf{k})=f_{0}(E)+\tau \frac{\partial f_{0}}{\partial E} \mathbf{v} \cdot \frac{\{\mathbf{F}+g \tau \mathbf{B} \times \mathbf{F}\}}{1+(g \tau B)^{2}}
$$

where we have set $\hbar=1$ and also from now on set $k_{B}=$ $c=1 . \mathbf{v}=\partial E / \partial \mathbf{k}, \tau$ is the relaxation time and we assume that it depends on the kinetic energy, $E=k^{2} / 2 m$. $\mathbf{F}=(E-\mu) \nabla T / T+\nabla(\mu-2 e \phi)$ and $g=g_{s}=2 e / m_{s}$ for singlet bipolarons with the energy $E=k^{2} /\left(2 m_{s}\right)$. For triplet bipolarons, $\mathbf{F}=(E+J-\mu) \nabla T / T+\nabla(\mu-2 e \phi)$, $g=g_{t}=2 e / m_{t}$ and the energy $E=k^{2} /\left(2 m_{t}\right) . \quad \mathbf{F}=$ $(E+\Delta / 2-\mu / 2) \nabla T / T+\nabla(\mu / 2-e \phi), E=k^{2} /\left(2 m_{p}\right)$ and $g=g_{p}=e / m_{p}$ for thermally excited polarons. Here $m_{s, t, p}$ are the singlet and triplet bipolaron and polaron masses of two-dimensional carriers.

Eqns.(7-11) are used to find the thermal and electrical currents induced by the applied thermal and potential gradients in a magnetic field.

\section{ELECTRICAL AND THERMAL CURRENTS}

The electrical current for each carrier is given by

$$
\mathbf{j}^{\alpha}=q \sum_{\mathbf{k}} \mathbf{v} f_{1}^{\alpha}(\mathbf{k})
$$

where $\alpha=s, p, t$, and $q$ is the carrier charge. We find the $\mathrm{x}$ direction component as

$$
\begin{aligned}
j_{x}^{\alpha}= & a_{x x}^{\alpha} \nabla_{x}(\mu-2 e \phi)+a_{x y}^{\alpha} \nabla_{y}(\mu-2 e \phi) \\
& +b_{x x}^{\alpha} \nabla_{x} T+b_{x y}^{\alpha} \nabla_{y} T
\end{aligned}
$$

and also the y direction component

$$
\begin{aligned}
j_{y}^{\alpha}= & a_{y y}^{\alpha} \nabla_{y}(\mu-2 e \phi)+a_{y x}^{\alpha} \nabla_{x}(\mu-2 e \phi) \\
& +b_{y y}^{\alpha} \nabla_{y} T+b_{y x}^{\alpha} \nabla_{x} T
\end{aligned}
$$

where

$$
\begin{aligned}
& a_{x x}^{p}=a_{y y}^{p}=\frac{e n_{p}}{2 m_{p}}\left\langle\tau_{p}\right\rangle, \\
& a_{y x}^{p}=-a_{x y}^{p}=\frac{e g_{p} B n_{p}}{2 m_{p}}\left\langle\tau_{p}^{2}\right\rangle, \\
& b_{x x}^{p}=b_{y y}^{p}=\frac{e n_{p}}{T m_{p}}\left\langle\tau_{p}\{E+\Delta / 2-\mu / 2\}\right\rangle, \\
& b_{y x}^{p}=-b_{x y}^{p}=\frac{e g_{p} B n_{p}}{T m_{p}}\left\langle\tau_{p}^{2}\{E+\Delta / 2-\mu / 2\}\right\rangle, \\
& a_{x x}^{s, t}=a_{y y}^{s, t}=\frac{2 e n_{s, t}}{m_{s, t}}\left\langle\tau_{s, t}\right\rangle, \\
& a_{y x}^{s, t}=-a_{x y}^{s, t}=\frac{2 e g_{s, t} B n_{s, t}}{m_{s, t}}\left\langle\tau_{s, t}^{2}\right\rangle, \\
& b_{x x}^{s}=b_{y y}^{s}=\frac{2 e n_{s}}{T m_{s}}\left\langle\tau_{s}\{E-\mu\}\right\rangle, \\
& b_{y x}^{s}=-b_{x y}^{s}=\frac{2 e g_{s} B n_{s}}{T m_{s}}\left\langle\tau_{s}^{2}\{E-\mu\}\right\rangle \\
& b_{x x}^{t}=b_{y y}^{t}=\frac{2 e n_{t}}{T m_{t}}\left\langle\tau_{t}\{E+J-\mu\}\right\rangle, \\
& b_{y x}^{t}=-b_{x y}^{t}=\frac{2 e g_{t} B n_{t}}{T m_{t}}\left\langle\tau_{t}^{2}\{E+J-\mu\}\right\rangle,
\end{aligned}
$$


and

$$
\left\langle\tau_{\alpha}^{r}\right\rangle=\frac{\int_{0}^{\infty} d E E \tau_{\alpha}^{r}(E)\left[1+\left(g_{\alpha} \tau_{\alpha}(E) B\right)^{2}\right]^{-1} \partial f_{0}^{\alpha} / \partial E}{\int_{0}^{\infty} d E f_{0}^{\alpha}}
$$

The number densities, $n_{\alpha}$ of the three carriers can be evaluated as

$$
\begin{gathered}
n_{p}=\frac{m_{p} T}{\pi} \ln \left[1+\exp \left(-\frac{\Delta-\mu}{2 T}\right)\right], \\
n_{s}=-\frac{m_{s} T}{2 \pi} \ln \left[1-\exp \left(\frac{\mu}{T}\right)\right], \\
n_{t}=-\frac{3 m_{t} T}{2 \pi} \ln \left[1-\exp \left(\frac{\mu-J}{T}\right)\right] .
\end{gathered}
$$

and the results are shown in Fig. 2 for optimally doped $\mathrm{YBa}_{2} \mathrm{Cu}_{3} \mathrm{O}_{6.95}$ using experimental estimates of the energy gaps as measured by Mihailovic et $\mathrm{al}^{28}$.

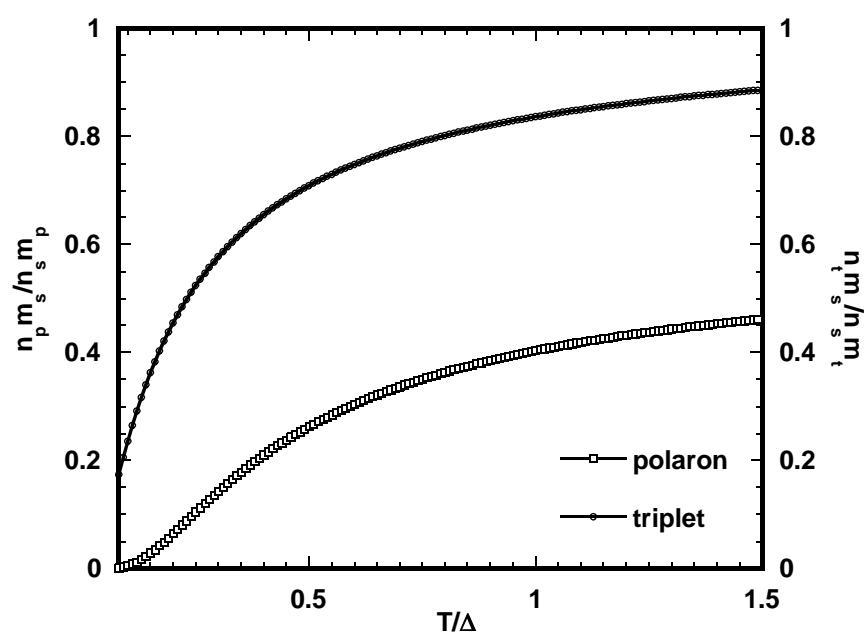

FIG. 2: number density ratios for triplets and polarons in the optimally doped regime

To find the thermal currents we calculate the energy flux for each carrier which are given by

$$
\mathbf{w}^{\alpha}=\sum_{\mathbf{k}} \mathbf{v} f_{1}^{\alpha}(\mathbf{k}) E^{\alpha}
$$

where $E^{p}=E+\Delta / 2+e \phi, E^{s}=E+2 e \phi$ and $E^{t}=$ $E+J+2 e \phi$. The x direction component is

$$
\begin{aligned}
w_{x}^{\alpha}= & c_{x x}^{\alpha} \nabla_{x}(\mu-2 e \phi)+c_{x y}^{\alpha} \nabla_{y}(\mu-2 e \phi) \\
& +d_{x x}^{\alpha} \nabla_{x} T+d_{x y}^{\alpha} \nabla_{y} T
\end{aligned}
$$

and the $\mathrm{y}$ direction component is

$$
\begin{aligned}
w_{y}^{\alpha}= & c_{y y}^{\alpha} \nabla_{y}(\mu-2 e \phi)+c_{y x}^{\alpha} \nabla_{x}(\mu-2 e \phi) \\
& +d_{y y}^{\alpha} \nabla_{y} T+d_{y x}^{\alpha} \nabla_{x} T
\end{aligned}
$$

where

$$
\begin{aligned}
& c_{x x}^{p}=c_{y y}^{p}=\frac{n_{p}}{2 m_{p}}\left\langle E^{p} \tau_{p}\right\rangle, \\
& c_{y x}^{p}=-c_{x y}^{p}=\frac{g_{p} B n_{p}}{2 m_{p}}\left\langle E^{p} \tau_{p}^{2}\right\rangle, \\
& d_{x x}^{p}=d_{y y}^{p}=\frac{n_{p}}{T m_{p}}\left\langle E^{p}(E+\Delta / 2-\mu / 2) \tau_{p}\right\rangle, \\
& d_{y x}^{p}=-d_{x y}^{p}=\frac{g_{p} B n_{p}}{T m_{p}}\left\langle E^{p}(E+\Delta / 2-\mu / 2) \tau_{p}^{2}\right\rangle, \\
& c_{x x}^{s}=c_{y y}^{s}=\frac{n_{s}}{m_{s}}\left\langle E^{s} \tau_{s}\right\rangle, \\
& c_{y x}^{s}=-c_{x y}^{s}=\frac{g_{s} B n_{s}}{m_{s}}\left\langle E^{s} \tau_{s}^{2}\right\rangle, \\
& d_{x x}^{s}=d_{y y}^{s}=\frac{n_{s}}{T m_{s}}\left\langle E^{s}(E-\mu) \tau_{s}\right\rangle, \\
& d_{y x}^{s}=-d_{x y}^{s}=\frac{g_{s} B n_{s}}{T m_{s}}\left\langle E^{s}(E-\mu) \tau_{s}^{2}\right\rangle, \\
& c_{x x}^{t}=c_{y y}^{t}=\frac{n_{t}}{m_{t}}\left\langle E^{t} \tau_{t}\right\rangle, \\
& c_{y x}^{t}=-c_{x y}^{t}=\frac{g_{t} B n_{t}}{m_{t}}\left\langle E^{t} \tau_{t}^{2}\right\rangle, \\
& d_{x x}^{t}=d_{y y}^{t}=\frac{n_{t}}{T m_{t}}\left\langle E^{t}(E+J-\mu) \tau_{t}\right\rangle, \\
& d_{y x}^{t}=-d_{y x}^{t}=\frac{g_{t} B n_{t}}{T m_{t}}\left\langle E^{t}(E+J-\mu) \tau_{t}^{2}\right\rangle .
\end{aligned}
$$

\section{TRANSPORT COEFFICIENTS}

In thermal transport experiments one has to make sure the electrical current is set to zero $\mathbf{j}=\mathbf{j}_{\mathbf{p}}+\mathbf{j}_{\mathbf{s}}+\mathbf{j}_{\mathbf{t}}=0$. Using eqns. (13) and (14), we find,

$$
\begin{aligned}
\nabla_{x}(\mu-2 e \phi)= & -\frac{b_{y x} a_{y x}+b_{x x} a_{x x}}{\left(a_{x x}\right)^{2}+\left(a_{y x}\right)^{2}} \nabla_{x} T \\
& +\frac{b_{y x} a_{x x}-b_{x x} a_{y x}}{\left(a_{x x}\right)^{2}+\left(a_{y x}\right)^{2}} \nabla_{y} T
\end{aligned}
$$

and

$$
\begin{aligned}
\nabla_{y}(\mu-2 e \phi)= & -\frac{b_{y y} a_{y y}+b_{y x} a_{y x}}{\left(a_{x x}\right)^{2}+\left(a_{y x}\right)^{2}} \nabla_{y} T \\
& +\frac{b_{x x} a_{y x}-b_{y x} a_{x x}}{\left(a_{x x}\right)^{2}+\left(a_{y x}\right)^{2}} \nabla_{x} T
\end{aligned}
$$

where $b_{x x}=b_{x x}^{p}+b_{x x}^{s}+b_{x x}^{t}, a_{y x}=a_{y x}^{p}+a_{y x}^{s}+a_{y x}^{t}$, etc. By substituting eqn (24) and (25) into eqn (21) and (22) we obtain for the thermal conductivities $\kappa_{x x}$, and for the thermal Hall conductivity, $\kappa_{y x}$

$$
\begin{aligned}
\kappa_{x x}= & d_{x x}-c_{x x} \frac{b_{y x} a_{y x}+b_{x x} a_{x x}}{\left(a_{x x}\right)^{2}+\left(a_{y x}\right)^{2}} \\
& +c_{y x} \frac{b_{y x} a_{x x}-b_{x x} a_{y x}}{\left(a_{x x}\right)^{2}+\left(a_{y x}\right)^{2}}
\end{aligned}
$$


and

$$
\begin{aligned}
\kappa_{y x}= & d_{y x}-c_{y x} \frac{b_{y x} a_{y x}+b_{x x} a_{x x}}{\left(a_{x x}\right)^{2}+\left(a_{y x}\right)^{2}} \\
& +c_{x x} \frac{b_{x x} a_{y x}-b_{y x} a_{x x}}{\left(a_{x x}\right)^{2}+\left(a_{y x}\right)^{2}}
\end{aligned}
$$

The electrical conductivity is defined in the presence of an electric field $\mathbf{E}=-\nabla \phi$ and in the absence of a thermal and chemical gradient, $\nabla T=\nabla \mu=0$,

$$
j_{x}=2 e a_{x x} E_{x}-2 e a_{y x} E_{y}
$$

and

$$
j_{y}=2 e a_{y y} E_{y}+2 e a_{y x} E_{x}
$$

which gives

$$
\begin{aligned}
& \sigma_{x x}=\sigma_{y y}=2 e a_{x x} \\
& \sigma_{y x}=-\sigma_{x y}=2 e a_{y x}
\end{aligned}
$$

We can combine eqns. (26),(27),(30) and (31) to obtain the Lorenz number and the Hall Lorenz number as

$$
\begin{aligned}
L= & \frac{e}{2 T a_{x x}}\left(d_{x x}-c_{x x} \frac{b_{y x} a_{y x}+b_{x x} a_{x x}}{\left(a_{x x}\right)^{2}+\left(a_{y x}\right)^{2}}\right. \\
& \left.+c_{y x} \frac{b_{y x} a_{x x}-b_{x x} a_{y x}}{\left(a_{x x}\right)^{2}+\left(a_{y x}\right)^{2}}\right) \\
L_{H}= & \frac{e}{2 T a_{y x}}\left(d_{y x}-c_{y x} \frac{b_{y x} a_{y x}+b_{x x} a_{x x}}{\left(a_{x x}\right)^{2}+\left(a_{y x}\right)^{2}}\right. \\
& \left.+c_{x x} \frac{b_{x x} a_{y x}-b_{y x} a_{x x}}{\left(a_{x x}\right)^{2}+\left(a_{y x}\right)^{2}}\right),
\end{aligned}
$$

respectively.

We also define the Hall ratio. The Hall effect is the appearance of an electric field perpendicular to both the current and applied magnetic field, which is perpendicular to the current. If the current flows in the x-direction only, $j_{x}=j$ and $j_{y}=0$, then we can eliminate $E_{x}$ and find the Hall ratio, which is defined as $R_{H}=E_{y} /(B j)$,

$$
R_{H}=\frac{a_{y x}}{2 e\left[\left(a_{x x}\right)^{2}+\left(a_{y x}\right)^{2}\right] B}
$$

\section{WEAK-FIELD APPROXIMATION}

If the magnetic field is weak, $g_{\alpha} \tau_{\alpha} B<<1$, we can ignore all terms in $B^{2}$ and higher order. The previous definition of $\left\langle\tau_{\alpha}^{r}\right\rangle$ simplifies and becomes

$$
\left\langle\tau_{\alpha}^{r}\right\rangle=\frac{\int_{0}^{\infty} E \tau_{\alpha}^{r} d E \partial f_{0}^{\alpha} / \partial E}{\int_{0}^{\infty} d E f_{0}^{\alpha}}
$$

Next we can apply the coefficients, eqns (15) and (23), into the transport coefficients derived in the previous section.
The resistivity, Hall resistivity and Hall ratio for a triplet, singlet bipolaron and polaron system are respectively

$$
\begin{aligned}
\rho_{x x} & =\rho_{y y}=\frac{m_{p}}{e^{2} n_{p}\left\langle\tau_{p}\right\rangle\left[1+4 A_{s 1}+4 A_{t 1}\right]} \\
\rho_{y x} & =-\rho_{x y}=\frac{m_{p}}{e^{2} g_{p} B n_{p}\left\langle\tau_{p}^{2}\right\rangle\left[1+4 A_{s 2}+4 A_{t 2}\right]} \\
R_{H} & =\frac{g_{p}\left\langle\tau_{p}^{2}\right\rangle m_{p}\left[1+4 A_{s 2}+4 A_{t 2}\right]}{e^{2}\left\langle\tau_{p}\right\rangle^{2} n_{p}\left[1+4 A_{s 1}+4 A_{t 1}\right]^{2}}
\end{aligned}
$$

where

$$
A_{s 1, t 1}=\frac{n_{s, t}\left\langle\tau_{s, t}\right\rangle m_{p}}{n_{p}\left\langle\tau_{p}\right\rangle m_{s, t}}
$$

and

$$
A_{s 2, t 2}=\frac{g_{s, t} n_{s, t}\left\langle\tau_{s, t}^{2}\right\rangle m_{p}}{g_{p} n_{p}\left\langle\tau_{p}^{2}\right\rangle m_{s, t}} .
$$

With the use of eqns.(32) and (33) we arrive at the Lorenz number

$$
\begin{aligned}
& L=D_{1}\left[L_{p}+4 A_{s 1} L_{s}+4 A_{t 1} L_{t}\right]+ \\
& D_{1}^{2}\left[A_{s 1}\left(2 \Gamma_{p}-\Gamma_{s}+\Delta / T\right)^{2}+\right. \\
& A_{t 1}\left(2 \Gamma_{p}-\Gamma_{t}+(\Delta-J) / T\right)^{2}+ \\
& \left.\left.4 A_{s 1} A_{t 1}\left(\Gamma_{t}-\Gamma_{s}+J / T\right)^{2}\right]\right\}
\end{aligned}
$$

and the Hall Lorenz number

$$
\begin{aligned}
L_{H}= & D_{2}\left(L_{p}+4 A_{s 2} L_{s}+4 A_{t 2} L_{t}\right)+D_{1} D_{2}^{2} \times \\
& {\left[\left(4 A_{s 1}^{2}+2 A_{s 2}\right)\left(\Delta / T+2 \Gamma_{p}-\Gamma_{s}\right)^{2}+\right.} \\
& 4 A_{t 1}^{2}\left(\left(J^{2}+\Delta^{2}\right) / T^{2}+\right. \\
& \left.\left(2 \Gamma_{p}-\Gamma_{t}\right)\left(2(\Delta-J) / T+2 \Gamma_{p}-\Gamma_{t}\right)\right)- \\
& 8 A_{t 1} A_{s 2}\left(\Delta / T+2 \Gamma_{p}-\Gamma_{s}\right)\left(J / T+\Gamma_{t}-\Gamma_{s}\right)+ \\
& 16\left(A_{t 1}^{2} A_{s 2}+A_{s 1}^{2} A_{t 2}\right)\left(J / T+\Gamma_{t}-\Gamma_{s}\right)^{2}+ \\
& 2 A_{t 2}\left(\left(\Delta / T+2 \Gamma_{p}-J / T\right)^{2}+\right. \\
& \left.\Gamma_{t}\left(1+2(J-\Delta) / T-4 \Gamma_{p}\right)\right)+ \\
& 8 A_{s 1} A_{t 2}\left(\Gamma_{s}\left((\Delta-J) / T-2 \Gamma_{t}\right)+\right. \\
& \left(J / T+\Gamma_{t}\right)\left((J-\Delta) / T+\Gamma_{t}\right)- \\
& \left.2 \Gamma_{p}\left(J / T+\Gamma_{t}-\Gamma_{s}\right)\right)+4 A_{s 1} A_{t 1} \times \\
& \left(8 \Gamma_{p}^{2}+\Gamma_{s}\left((J-2 \Delta) / T+\Gamma_{t}\right)-2(\Delta / T) \times\right. \\
& \left.\left.\left((J+\Delta) / T+\Gamma_{t}\right)-4 \Gamma_{p}\left(J / T-\Gamma_{s}+\Gamma_{t}-2 \Delta / T\right)\right)\right]
\end{aligned}
$$

for a strongly-coupled electron-phonon system in the bipolaronic regime, where we have introduced the dimensionless parameters

$$
\Gamma_{\alpha}=\frac{\int_{0}^{\infty} d E E^{2} \tau_{\alpha}(E) \partial f_{0}^{\alpha} / \partial E}{T \int_{0}^{\infty} d E E \tau_{\alpha}(E) \partial f_{0}^{\alpha} / \partial E}
$$

$$
\gamma_{\alpha}=\frac{\int_{0}^{\infty} d E E^{3} \tau_{\alpha}(E) \partial f_{0}^{\alpha} / \partial E}{T^{2} \int_{0}^{\infty} d E E \tau_{\alpha}(E) \partial f_{0}^{\alpha} / \partial E}
$$


and $D_{1,2}=\left(1+4 A_{s 1,2}+4 A_{t 1,2}\right)^{-1}$. Also

$$
\begin{aligned}
L_{p} & =\left(\gamma_{p}-\Gamma_{p}^{2}\right), \\
L_{s, t} & =\left(\gamma_{s, t}-\Gamma_{s, t}^{2}\right) / 4 .
\end{aligned}
$$

In the limit of a pure polaronic system (i.e. $A_{t}=A_{s}=$ 0 ) the Lorenz number and Hall Lorenz number is

$$
L=L_{H}=L_{p} .
$$

If polarons are degenerate then $L_{p}=L_{0}=\pi^{2} / 3$ for any elastic scattering and any dimensionality. Indeed for a majority of metals $L$ falls in the region of approximately $(3.1-3.3)$ as expected in a degenerate Fermi liquid. However $L_{p}$ depends on the dimensionality and the scattering mechanism for non-degenerate polarons. In the opposite limit we have a pure singlet or triplet bipolaronic system (i.e. $A_{t}=A_{p}=0$ or $A_{s}=A_{p}=0$ ). We obtain from eqns.(41) and (42)

$$
L=L_{H}=L_{s, t}
$$

respectively, which is about 6 times smaller than $L_{0}$. The last equation is expected for a charged Bose gas as noted by Mott and one of the authors (ASA $)^{36}$. In the general case our final equations, eqn.(41) and (42), yield Lorenz numbers that differ significantly from both limits at finite temperatures. The main difference originates from the extra terms, which describe an interference of polaron and bipolaron contributions to the heat flow. In the lowtemperature regime, $T \ll J$ and $\Delta$, this contribution is exponentially small because the densities of triplet pairs and single polarons are small. However, this contribution becomes important in the intermediate temperature range $T_{c}<T<T^{*}$ because of the combination of the factors $(J / T)^{2}$ and $(\Delta / T)^{2}$ with the exponential form of the number densities. The contribution appears as the result of the recombination of a pair of polarons into triplet and singlet bound states at the cold end of the sample, which is reminicent to the contribution of the electron-hole pairs to the heat flow in semiconductors 53 . Our expressions for the Lorenz numbers eqns. $(41,42)$ anticipate its values to deviate from $L_{0} / 4$ for our system of predominantly charged Bose particles. Here the $\Gamma$ terms are representative of a type of scattering mechanism(s), which can be a combination of types. In the non-degenerate system it is given by

$$
\left(\gamma-\Gamma^{2}\right)=\Gamma=(r+2)
$$

where $r$ is related to the energy dependence of the scattering time

$$
\tau \propto E^{r} .
$$

\section{NUMERICAL ANALYSIS AND THE PHONON THERMAL CONDUCTIVITY}

Here we have shown that the present model fits the Hall Lorenz number, $L_{H}$, measured by Zhang et al $\stackrel{42}{~}$.

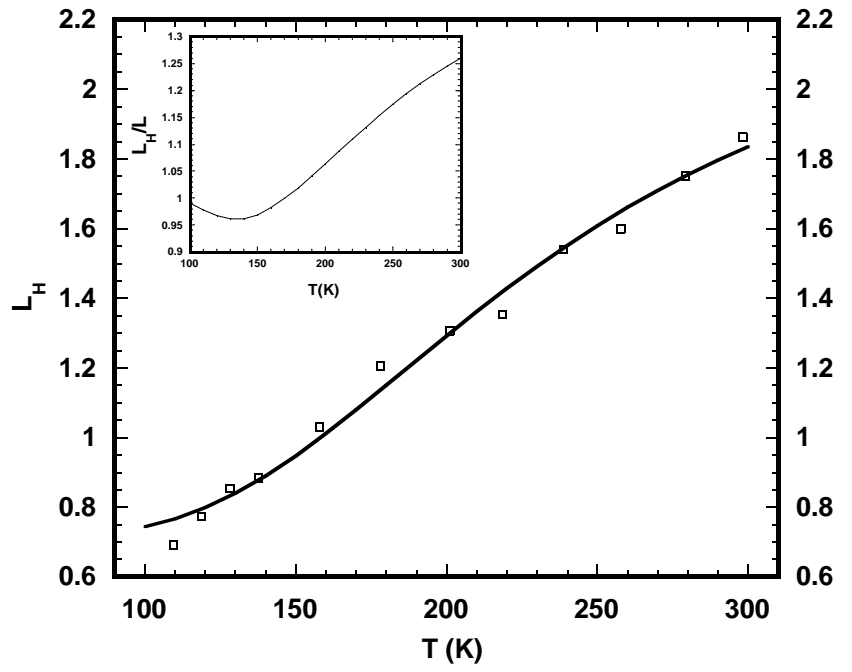

FIG. 3: $L_{H}$ which fits nicely to experiment $\left(\mathrm{YBa}_{2} \mathrm{Cu}_{3} \mathrm{O}_{6.95}\right)$ using $\Theta_{b} / \Theta_{p}=0.36$. This experiment showed a clear violation of the W-F law. The inset gives the ratio of Hall Lorenz number to Lorenz number

From these fits the phonon thermal conductivity can also be extracted.

The charge and spin gap value was estimated by Mihailovic et al. 28 for optimally doped $\mathrm{YBa}_{2} \mathrm{Cu}_{3} \mathrm{O}_{6+x}$ (x is the number of doped oxygen ions), giving $\Delta / 2=675 \mathrm{~K}$ and $J=150 K$, in their systematic analysis of charge and spin spectroscopies. According to Ref 31 the main scattering mechanism above $T_{c}$ is the particle-particle collisions which gives a relaxation time $\tau_{s, t, p} \propto 1 / T^{2}$. The chemical potential is pinned near the mobility edge, so that $y=\exp (\mu / T) \approx 0.6$ in a wide temperature range, if the number of localised states in the random potential is about the same as the number of bipolarons 31 . In $\mathrm{YBa}_{2} \mathrm{Cu}_{3} \mathrm{O}_{6+x}$ every excess oxygen ion $x$ can localise a bipolaron so this approximation is reasonable. As a result, there is only one fitting parameter in $L_{H}$, Eq.(42) which is the ratio of the bipolaron and polaron Hall angles $\Theta_{b} / \Theta_{p}$, where $\Theta_{\alpha}=q B \tau_{\alpha} / m_{\alpha}$ and we assume $\Theta_{s}=\Theta_{t}=\Theta_{b}$.

The model gives a good fit, as shown in Fig.3, with a reasonable value of $\Theta_{b} / \Theta_{p}=0.36$. By using the same single parameter for $L$, as was used to fit $L_{H}$ to the experimental data, we can see that the ratio of the Lorenz numbers varies with temperature with $L$ being larger at lower temperatures and $L_{H}$ growing at higher temperatures (inset in fig. 3).

The model also describes the (quasi) linear in-plane resistivity, Hall resistivity $T^{2}$-dependence and the Hall ratio, as observed in the cuprates (fig 4). As mentioned earlier the extraction of the phonon thermal conductivity from experiment has proven difficult and inconclusive. Here we can extract this quantity by using our theoretically calculated $L$ (lower inset of fig. 5) and using experimental resistivity data 54 (upper inset of fig. 5). This 


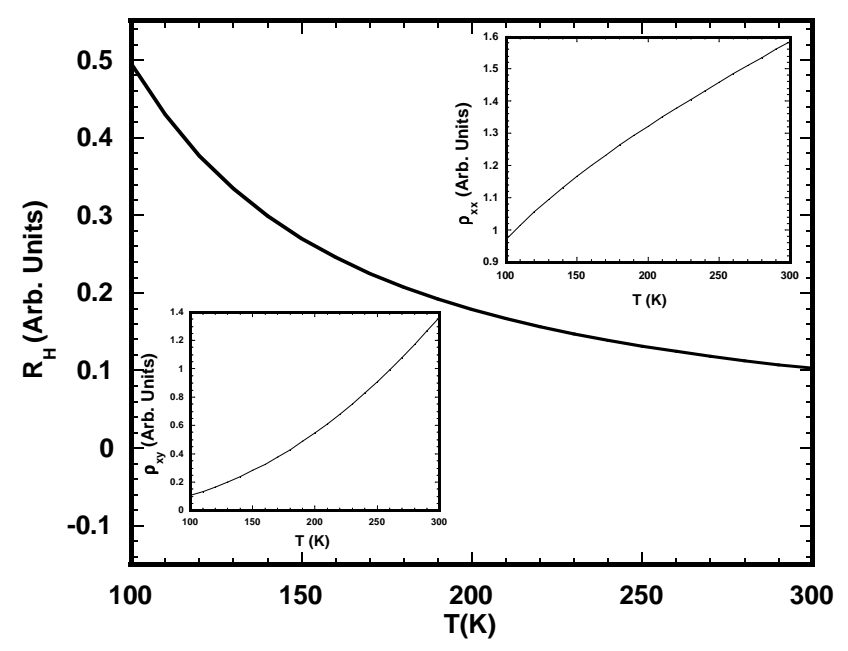

FIG. 4: T dependence of $\rho_{x x}, \rho_{x y}$ and $R_{H}$ for optimally doped cuprates.

allows us to find $\kappa_{e l}$ in the normal state, fig. 5. Using the same parameters as for the $L_{H}$ fittings, $L$ is seen to violate the $\mathrm{W}-\mathrm{F}$ law (lower inset in fig. 5). One can see that the electronic thermal conductivity is therefore very weakly T-dependent. These results are similar to the findings by Takenaka et al and Salamon et al. To find

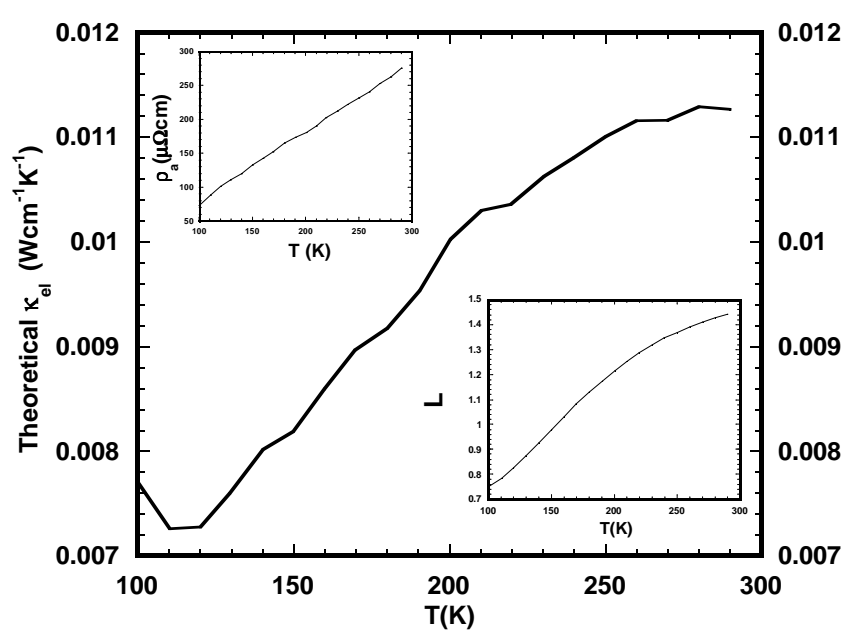

FIG. 5: Normal state $\kappa_{e l}(=L T / \rho)$ in optimally doped cuprates calculated using the theoretical $L$ (lower inset) and experimental resistivity data (upper inset).

$\kappa_{p h}$ we take for example Minami and Cooper ${ }^{55}$ thermal conductivity data on $\mathrm{YBa}_{2} \mathrm{Cu}_{3} \mathrm{O}_{6.93}$ and subtract our $\kappa_{e l}$. The results are shown in fig. 6 implying that the T-dependence of $\kappa$ is predominately due to the phonon contribution. We believe that this method is the only way to extract $\kappa_{p h}$ reliably. The lattice contribution to the diagonal heat flow appears to be much higher than it is anticipated in the framework of any Fermi-liquid model.

\section{CONCLUSIONS}

Recent measurements by Proust et $\mathrm{al}^{156}$ on $\mathrm{Tl}_{2} \mathrm{Ba}_{2} \mathrm{Cu} 0_{6+\delta}$ have suggested that the WiedemannFranz law holds perfectly well in the overdoped region and therefore conclude that the Fermi-liquid prevails at this doping. Alexandrov and Mott ${ }^{2}$, suggested that there might be a crossover from Bose-Einstein condensation to a BCS-like superconductivity across the phase diagram. Thus the results of Proust et al are compatible with the bipolaron picture. If the Fermi liquid does exist at overdoping then it is likely that the heavy doping causes an "overcrowding effect" where the polarons find it difficult to form bipolarons due to the larger number of competing holes ${ }^{2}$. Here we have shown that by the

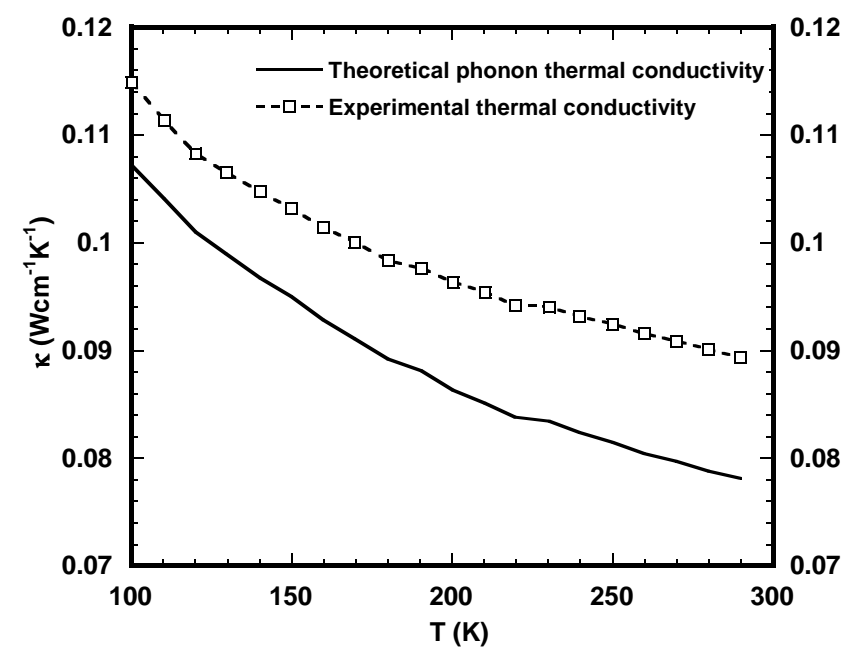

FIG. 6: Deduced $\kappa_{p h}$ and total $\kappa$ of Minami et al thermal conductivity data on $\mathrm{YBa}_{2} \mathrm{Cu}_{3} \mathrm{O}_{6.93}$ in the normal state.

necessary inclusion of thermally excited polarons and triplets as the temperature rises, the bipolaronic gas can predict the Hall Lorenz number as found experimentally. The fits here have been on optimally doped cuprates, however it is most likely that this model will work in the underdoped as well. Also this analysis, along with experimental data, allowed us to estimate the phonon contribution to the thermal conductivity which has remained elusive in recent years. The interference of the polaron and bipolaron contributions to the energy flow breaks down the Wiedemann-Franz law and results in the unusual temperature dependence of the Lorenz numbers. This work further validates the Bipolaronic model and it is our belief that the superconducting state heat transport can be described by it as well.

This work was supported by the Leverhulme Trust (grant F/00261/H) and by the EPSRC UK (grant R46977). We would like to thank Y. Zhang and P.W. Anderson for helpful discussions of some experimental and theoretical aspects. 
1 S. Chakravarty, R.B. Laughlin, D.K. Morr and C. Nayak, Phys. Rev. B, 63, 094503 (2001).

2 A.S. Alexandrov and N.F. Mott, High Temperature Superconductors and Other Superfluids, (Taylor and Francis, London, 1994).

${ }^{3}$ V.J. Emery and S.A. Kivelson, Nature, 374, 434 (1995).

4 P.W. Anderson, The Theory of Superconductivity in the High $T_{c}$ Cuprates, (Princeton University Press, Princeton, 1997).

5 S.C. Zhang, Science, 275, 4126 (1997).

6 D. Pines, A.V. Balatsky and P. Monthoux, Phys. Rev. Lett, 67, 3448 (1991).

7 G. Zhao, M.B. Hunt, H. Keller and K.A. Muller, Nature, 385, 236 (1997).

8 D. Mihailovic, C.M. Foster, K. Voss and A.J. Heeger, Phys. Rev. B42, 7989 (1990).

${ }^{9}$ P. Calvani, M.Capizzi, S. Lupi, P. Maselli, A. Paolone, P. Roy, S-W Cheong, W. Sadowski and E. Walker, Solid State Commun. 91, 113 (1994).

10 T. Timusk, C.C. Homes and W. Reichardt, in Anharmonic properties of High $T_{c}$ Cuprates, eds. D.Mihailovic, G. Ruani, E. Kaldis and K.A. Muller, 171(World Scientific, Singapore, 1995).

11 J.L. Cohn, S. Wolf and T.A. Vanderah, Phys. Rev. B , 45, 511 (1992).

12 T. Egami, J. Low Temp. Phys. 105, 791(1996).

13 A. Lanzara, P.V. Bogdanov, X.J. Zhou, S.A. Kellar, D.L. Feng, E.D. Lu, T. Yoshida, H. Eisaki, A. Fujimori,K. Kishio, J.I. Shimoyana, T. Noda, S. Uchida, Z. Hussain and Z.X. Shen, Nature, 412, 510 (2001).

14 A. Chainani, T. Yokoya, T. Kiss, S. Shin, T. Nishio and H. Uwe, Phys. Rev. B, 64, 180509 (2001).

15 G.M. Eliashberg, Sov.Phys. JETP, 11, 696 (1960).

16 A.S. Alexandrov, Phys. Rev. B, 46, 2838 (1992).

17 I.G. Lang and Y.A. Firsov, Sov. Phys. JETP, 16, 1301 (1963).

18 A.S. Alexandrov, Russ. J. Phys. Chem., 57, 273 (1983).

19 A.S. Alexandrov and E.A. Mazur, Zh. Eksp. Teor. Fiz., 96, 1773 (1989).

20 J.G. Bednorz and K.A. Muller, Angew. Chem., Int. Ed. Engl. 57, 735 (1988).

21 A.S. Alexandrov, Phys. Rev. B, 53, 2863 (1996).

22 C.R.A. Catlow, M.S. Islam and X. Zhang, J. Phys.:Condensed Matter, 10 No. 3, L49 (1998).

23 J. Bonca J and S.A. Trugman, Phys. Rev. B 64, 094507 (2001).

24 A.S. Alexandrov and P.E. Kornilovitch, J. Phys.:Condensed Matter, 14 No. 21, 5337 (2002).

25 A.S. Alexandrov and N.F. Mott, J. Supercond (US), 7, 599 (1994).

26 J. Rossat-Mignod, L.P. Regnault, P. Bourges, C. Vettier, P. Burlet and J.Y. Henry, Physica Scripta, 45, 74 1992).

27 H.A. Mook and M. Yethiraj, Phys. Rev. Lett, 70, 3490 (1993).

28 D. Mihailovic, V.V. Kabanov, K. Zagar, and J. Demsar,
Phys. Rev. B60, 6995 (1999) and references therein.

29 G. Deutscher, Nature, 397, 410 (1999).

30 A.S. Alexandrov and A.F. Andreev, EuroPhys. Lett., 54, 373 (2001).

31 A.S. Alexandrov, A.M. Bratkovsky and N.F. Mott, Phys. Rev. Lett, 72, 1734 (1994).

32 A.S.Alexandrov, A.M. Bratkovsky, N.F. Mott and E.K.H Salje, Physica C, 215, 359 (1993).

33 K.A. Muller, G.M. Zhao, K. Conder and H. Keller, J. Phys. Cond. Mat., 10, L291 (1998).

34 A.S. Alexandrov, Physica C, 305, 46 (1998).

35 A.S. Alexandrov, Phys. Rev. B, 46, 14932 (1992).

36 A.S. Alexandrov and N.F. Mott, Phys. Rev. Lett, 71, 1075 (1993).

37 A.S. Alexandrov, Doctoral thesis, Moscow Engineering Physics institute (1984)

38 A.S.Alexandrov and C.J. Dent, Phys. Rev. B, 60, 15414 (1999).

39 A. Junod, Studies of High-Temperature Superconductors Vol. 19, eds. A. Narlikar, 1 (Nova science, Commack, NY, 1996).

40 A.S. Alexandrov, W.H. Beere, V.V. Kabanov and W.Y Liang, Phys. Rev. Lett., 79, 1551 (1997).

41 A. Junod, A. Erb and C Renner, Physica C, 317-318, 333 (1999).

42 Y. Zhang, N.P. Ong, Z.A. Xu, K. Krishana, R. Gagnon, and L. Taillefer, Phys. Rev. Lett., 84, 2219 (2000).

43 K.K. Lee, A.S. Alexandrov and W.Y Liang, cond-mat/0211665 (2002).

44 K. Takenaka, Y. Fukuzumi, K. Mizuhashi, S. Uchida, H. Asaoka and H. Takei, Phys. Rev. B, 56, 5654 (1997).

45 R.C. Yu, M.B. Salamon, J.P. Lu and W.C. Lee, Phys. Rev. Lett., 69, 1431 (1992).

46 R.W. Hill, C. Proust, L. Taillefer, P. Fournier and R.L. Greene, Nature, 414, 711 (2001).

47 J. Takeya, Y. Ando, S. Komiya and X.F. Sun, cond-mat/0108055 (2002).

48 E. W. Carlson, V. J. Emery, S. A. Kivelson, and D. Orgad, cond-mat/0206217 and references therein.

49 A. J. Leggett, Physica Fennica 8, 125 (1973); J Stat. Phys. 93, 927 (1998);

V. N. Popov, Functional Integrals and Collective Excitations (Cambridge: Cambridge University Press) (1987).

50 A.S. Alexandrov, Physica C 363, 231 (2001).

51 K.A. Muller, Phil. Mag. Lett, 82, 279 (2002).

52 G.M. Zhao, Phys. Rev. B, 64, 024503 (2001).

53 A. Anselm, Introduction of Semiconductor Theory, (Prentice and Hall, New Jersey, 1981).

54 Kouji Segawa and Yoichi Ando, Phys. Rev. Lett., 86, 4907 (2001).

55 H. Minami and J.R. Cooper, Private communication.

56 C. Proust, E. Boakin, R.W. Hill, L. Taillefer and A.P. Mackenzie, cond-mat/0202101 (2002). 